\title{
Ginsenoside Rg1 reduces aldosterone-induced autophagy via the AMPK/mTOR pathway in NRK-52E cells
}

\author{
LI WANG $^{1,2 *}$, NAN MAO ${ }^{3 *}$, RUI-ZHI TAN ${ }^{2}$, HONG-LIAN WANG ${ }^{2}$, JI WEN $^{4}$, \\ YU-HANG LIU ${ }^{2}$, MD FURHAD $^{5}$ and JUN-MING FAN ${ }^{1,5}$ \\ ${ }^{1}$ State Key Laboratory of Biotherapy of Human Disease, West China Hospital, Sichuan University, Chengdu, Sichuan 610041; \\ ${ }^{2}$ Research Center of Combine Traditional Chinese and Western Medicine, Affiliated Traditional Medicine Hospital, \\ Luzhou Medical College, Luzhou, Sichuan 646000; ${ }^{3}$ Department of Nephrology, The First Affiliated Hospital \\ of Chengdu Medical College, Chengdu, Sichuan 610000; ${ }^{4}$ Department of Immunology and Rheumatology, \\ West China Hospital, Sichuan University, Chengdu, Sichuan 610041; ${ }^{5}$ Department of Nephrology, \\ The Affiliated Hospital of Luzhou Medical College, Luzhou, Sichuan 646000, P.R. China
}

Received March 9, 2015; Accepted May 20, 2015

DOI: $10.3892 /$ ijmm.2015.2242

\begin{abstract}
Aldosterone is a steroid hormone secreted from the adrenal cortex, which regulates blood pressure. Higher concentrations of aldosterone can cause several diseases, including hypertension, diabetic nephropathy and chronic kidney disease. Previous reports have demonstrated that aldosterone has a pathogenic role in renal injury via reactive oxygen species (ROS), which involves the regulation of autophagy. However, whether aldosterone can induce autophagy in renal tubular cells remains to be elucidated. In the present study, elevated autophagy was observed in rat renal tubular NRK-52E cells exposed to aldosterone, which was demonstrated by the increased number of autophagosomes, conversion of LC3-I to LC3-II and the expression of Beclin-1. The enhanced autophagy was accompanied by increased production of intracellular ROS, which was reversed by $\mathrm{N}$-acetylcysteine, a specific inhibitor of ROS signaling. Furthermore, treatment with ginsenoside Rg1 reduced the aldosterone-induced autophagy and production of ROS, possibly through reducing the phosphorylation of AMPK and preserving mTOR activity. These findings demonstrated that aldosterone promoted ROS generation and increased autophagy in the NRK-52E cells. Ginsenoside Rg1 effectively relieved aldosterone-induced oxidative stress and
\end{abstract}

Correspondence to: Professor Jun-Ming Fan, State Key Laboratory of Biotherapy of Human Disease, West China Hospital, Sichuan University, No. 17, The 3rd Segment of Renmin South Road, Wuhou, Chengdu, Sichuan 610041, P.R. China

E-mail: junmingfanlz@163.com

${ }^{*}$ Contributed equally

Key words: ginsenoside Rg1, NRK-52E cells, autophagy, aldosterone, AMPK abnormal autophagy, suggesting that $\operatorname{Rg} 1$ may be used as a potential therapeutic drug to inhibit the renal injury, which is induced by aldosterone.

\section{Introduction}

Aldosterone is a mineralocorticoid, which regulates electrolyte transport through epithelial mineralocorticoid receptors (1). There has been increased attention on the role of aldosterone in the renal injury. For several years, aldosterone was considered to execute its function only at distal fragments of renal tubules, whereas, subsequent investigation has revealed functional targets additional to the distal renal tubule, including proximal tubular cells, mesangial cells and podocytes (2). Several experimental data have demonstrated that aldosterone is involved in the progression of renal injury by triggering the production of reactive oxygen species (ROS), resulting in the apoptosis of tubular cells $(3,4)$. Specifically, aldosterone has been reported to promote the expression of nicotinamide adenine dinucleotide phosphate oxidase in renal tissues, and generate ROS to induce glomerular injury (5). This can be inferred from evidence that aldosterone promotes podocyte injury via the generation of ROS (6). In normal conditions, the balance of ROS generation is maintained by the antioxidant defense system, including superoxide dismutase (SOD) and catalase. However, when the balance is broken, DNA and proteins are oxidized by excessive ROS, accompanied by damage to organelles (7). In this case, autophagy is triggered to degrade the oxidized DNA, proteins and damaged organelles.

Autophagy is highly conserved between yeasts and humans, and is involved in the degradation of long-lived proteins and damaged organelles (8). It has been described as single- or double-membraned vesicles that contain cytoplasm, including organelles, at various stages of digestion (9). Autophagy in mammals can be stimulated by stress, including starvation and pathology, or by pharmacological agents, including rapamycin (10). It is also triggered by ROS, resulting from incom- 
plete reduction of ROS (11). In addition, the induced autophagy can be inhibited by antioxidant drugs, including $\mathrm{N}$-acetylcysteine (NAC), catalase, glutathione, melatonin and vitamin E (9).

In mammalian cells, several autophagy genes are involved in the formation of autophagosomes, including microtubule-associated protein 1 light chain 3 (MAP1-LC3; LC3), phosphatidylinositol 3-kinase and beclin-1 (12). LC3-I is the cytosolic form of LC3 and, during autophagy, LC3-I is modified to the membrane-bound form (LC3-II). Thus, the level of LC3-II is considered an early marker for the formation of autophagosomes (13). Vps34 is part of the autophagy-regulating macromolecular complex (PI3K complex), consisting of Beclin-1/Atg6, Atg14/barkor and p150/Vps15. The activity of Vps34 is increased by interaction with Beclin-1, suggesting that increasing the expression of Beclin-1 may enhance autophagy (14-17). Cells treated with pharmacological agents, including 3-methyladenine (3-MA) and chloroquine can inhibit autophagy (18). Dysfunctional autophagy has been reported in several human diseases, suggesting that decreased dysfunctional autophagy may relieve these diseases, however, whether autophagy can be induced by aldosterone in renal tubular cells and, if so, whether aldosterone-induced autophagy can be relieved by certain drugs, remains to be elucidated.

Ginsenoside-Rg1 ( $\mathrm{Rg} 1)$ is the major pharmacological active component of ginseng, which is a widely used traditional Chinese medicine (19). It has extensive pharmacological activities, including antioxidant, anti-inflammatory and anticancer properties $(20,21)$. Previous investigation has demonstrated that ginsenoside $\mathrm{Rg} 1$ enhances antioxidative protection and intracellular calcium homeostasis in a cardiomyocyte hypoxia/reoxygenation model (22), and can also inhibit autophagy in cardiomyocytes exposed to hypoxia/reoxygenation (23). Our previous study reported the effects of Rg1 in the protection of mouse podocytes from aldosterone-induced injury by inhibiting ROS generation (24). Based on the properties of $\operatorname{Rg} 1$ on relieving autophagy in several types of cell, it was hypothesized that Rg1 may also promote antioxidative protection in renal tubular cells to inhibit autophagy.

The present study was perforned to investigate the potential for aldosterone to induce autophagy in NRK-52E cells and to examine the effects of ginsenoside-Rg1 on relieving aldosterone-induced autophagy. In addition, the molecular mechanism underlying aldosterone-induced autophagy and Rg1-mediated therapy was investigated.

\section{Materials and methods}

Cell culture. The NRK-52E rat renal tubular cell line was purchased from the Type Culture Collection of the Chinese Academy of Sciences (Shanghai, China). The cells were cultured in Dulbecco's modified Eagle's medium (DMEM; GE Healthcare Life Sciences, Logan, UT, USA) supplemented with $5 \%$ fetal bovine serum (FBS; GE Healthcare Life Sciences), $100 \mathrm{U} / \mathrm{ml}$ penicillin and $100 \mathrm{mg} / \mathrm{ml}$ streptomycin at $37^{\circ} \mathrm{C}$ in $5 \% \mathrm{CO}_{2}$ air, conditioned at $100 \%$ humidity.

Drug treatment. The NRK-52E cells were seeded in a 6-well plate at a density of 0.5 million cells/well followed by drug treatment the following day. The NRK-52E cells were incubated with aldosterone $\left(10^{-8} \mathrm{M}\right)$, NAC $(50 \mathrm{mM})$, chloroquine
(CL; $10 \mu \mathrm{M})$, 3-Methyladenine (3-MA; $14 \mathrm{mM}$ ) and rapamycin $(1 \mathrm{ng} / \mathrm{ml})$, respectively. All the above drugs were purchased from Sigma-Aldrich (St. Louis, MO, USA). To inhibit autophagy, the cells were pre-incubated with ginsenoside Rg1 (Rg1; $80 \mathrm{ng} / \mathrm{ml})$ for $2 \mathrm{~h}$ (at $37^{\circ} \mathrm{C}$ ), following which aldosterone was added to the culture for up to $12 \mathrm{~h}$. Rg1 (>98\% pure) was purchased from DiDa Kexiang Biological Co., Ltd. (Guizhou, China). Cells treated with fresh medium only were used as the controls.

Transmission electron microscopy (TEM). For the analysis of autophagosomes using transmission electron microscopy (TEM), following treatment, the cells were washed briefly with phosphate-buffer saline (PBS) and fixed with 3\% glutaraldehyde (Sangon Biotech, Shanghai, China) followed by post-fixation with $1 \% \mathrm{OsO}_{4}$ (Sangon Biotech). The sample was then dehydrated in acetone and embedded in Epon 812 (Nissin EM, Tokyo, Japan). Ultra-thin sections were prepared and stained with $2.0 \%$ uranyl acetate/lead citrate, which were further visualized using a Hitachi H-600IV electron microscope (Hitachi, Ltd., Tokyo, Japan).

Western blot analysis. For total protein extraction, the cells were rinsed with ice-cold PBS containing $5 \mathrm{mM}$ EDTA and lysed with radioimmunoprecipitation assay lysis buffer $(20 \mu \mathrm{l}$; Sangon Biotech) containing $1 \mathrm{mM}$ phenylmethylsulfonyl fluoride (Beyotime Biotech, Beijing, China) on ice. Following removal of cellular debris by centrifugation (at 13,000 rpm at $4^{\circ} \mathrm{C}$ for $15 \mathrm{~min}$ ), the protein concentration was determined using a bicinchoninic acid protein assay kit (Nanjing KeyGen Biotech Co., Ltd., Nanjing, China). Total protein $(25 \mu \mathrm{g})$ was resolved on an $10 \%$ SDS-PAGE gel (Solarbio, Beijing, China) and transferred onto a polyvinylidene difluoride membrane (EMD Millipore, Billerica MA, USA). Following blocking with $5 \%$ milk, the blot was probed with the indicated primary antibodies at $4^{\circ} \mathrm{C}$ overnight, followed by washing with Tris-buffered saline with Tween 20 three times for 5 min each time. The blots were then incubated with the corresponding secondary antibody, to recognize the primary antibody, at room temperature for $1 \mathrm{~h}$. Following washing, as above, the signal was developed using Immobilon Western Chemiluminescent HRP substrate (EMD Millipore) and exposed to X-ray film (Kodak, Rochester, NY, USA). The primary antibodies used in the present study were as follows: LC3-II (\#2775, polyclonal, rabbit anti-mouse, 1:1,000; Cell Signaling Technology, Inc., Danvers, MA, USA), beclin-1 (\#3495S, polyclonal, rabbit anti-mouse, 1:1,000; Cell Signaling Technology, Inc.), SOD2 (\#2299-1, polyclonal, rabbit anti-mouse, 1:2,000; Epitomics, Burlingame, CA, USA) and catalase (\#8841, polyclonal, rabbit anti-mouse, 1:1,000; Cell Signaling Technology, Inc.) and the secondary antibodies were horseradish peroxidase-conjugated secondary anti-mouse or anti-rabbit antibodies (\#ZB2301, goat anti-rabbit, 1:5,000; ZSGB-Bio, Beijing, China).

Immunofluorescence. For immunofluorescence, the NRK-52E cells, grown on coverslips, were harvested at indicated time-points and fixed with $4 \%$ paraformaldehyde (Sangon Biotech) at room temperature following a brief rinse with PBS. The cells were then washed twice with PBS, permeablized with $2.5 \%$ Triton X-100 (Sangon Biotech), blocked with 10\% goat serum in PBS and incubated with primary rabbit polyclonal 
anti-LC3B antibody (\#2775, polyclonal, rabbit anti-mouse, 1:400; Cell Signaling Technology, Inc.) overnight at $4^{\circ} \mathrm{C}$ in a humidified chamber. Subsequently, the cells were incubated with Alexa Fluor ${ }^{\circledR}$ 488-conjugated donkey anti-rabbit IgG (H+L) secondary antibody (\#A21206, 1:400; Invitrogen Life Technologies, Carlsbad, CA, USA) and rhodamine-labeled phalloidin (\#R415, 1:1,000; Invitrogen Life Technologies) at room temperature for $1 \mathrm{~h}$. The nuclei of the cells were visualized using 4',6-diamino-2-phenylindole (DAPI) staining. Finally, immunofluorescence images were captured using a Nikon Eclipse 50i microscope (Nikon) and the number of green fluorescent puncta in 50 cells/group were counted..

Acridine orange staining. The NRK-52E cells were grown on coverslips to $40 \%$ density, and treated with the indicated drug combinations, described above, for the indicated period of time. The cell medium was replaced with fresh medium containing acridine orange $(0.05 \mathrm{~g} / \mathrm{ml}$; Sigma-Aldrich) and Hoechst 33342 ( $1 \mu \mathrm{g} / \mathrm{ml}$; Invitrogen Life Technologies) and incubated for a further $15 \mathrm{~min}\left(\right.$ at $37^{\circ} \mathrm{C}$ ) in the cell culture incubator. Following brief washing, the cells were fixed with $4 \%$ paraformaldehyde at room temperature for $10 \mathrm{~min}$. The cells were subjected a to additional washing in PBS for $5 \mathrm{~min}$, and the corresponding coverslip was mounted to a glass slide. Images of the cells were captured using a Nikon Eclipse 50i microscope (Nikon).

Measurement of intracellular levels of ROS. Intracellular ROS was measured using a 2,7-dichlorofluorescin diacetate (DCFH-DA) assay, which was performed according to the manufacturer's instructions (Nanjing KeyGen Biotech). Briefly, $5 \mu$ l DCFH-DA (1 mM) was added to each well of a 6-well plate; Rosup $(1: 1,000)$ was added to the well as a positive control of ROS, and the cells were cultured for $30 \mathrm{~min}$ at $37^{\circ} \mathrm{C}$ in the dark. Following washing three times with PBS, the cells were analyzed using a flow cytometer (FACSAria; BD Biosciences, San Jose, CA, USA) and data were processed using the CellQuest program (version 3.2.1; BD Biosciences).

Data analysis. Data are presented as the mean \pm standard deviation. Multiple groups of quantitative data were compared using analysis of variance. Data were analyzed using SPSS software 13.0 (SPSS, Inc., Chicago, IL, USA). P<0.05 was considered to indicate a statistically significant difference.

\section{Results}

Aldosterone promotes autophagy in NRK-52E cells. To investigate the formation of autophagy in aldosterone-treated NRK-52E cells in the present study, TEM was used to observe the ultrastructural changes. This observation revealed that certain morphological changes had appeared, including the formation of double- and multiple-membrane encapsulated portions of the cytoplasm and/or organelles (autophagosomes) (Fig. 1A). Increased numbers of autophagosomes were observed in the aldosterone-treated NRK-52E cells, compared with the control cells (Fig. 1B). In order to further confirm that the autophagy in the NRK-52E cells was induced by aldosterone, immunofluorescence analysis was performed. The NRK-52E cells were treated with anti-LC3B antibody, following which the green fluorescent puncta, which represented autophagosomes, were examined under fluorescence microscopy (Fig. 1C). Prior to antibody treatment, the cells were pre-incubated with either buffer (control), aldosterone, rapamycin, rapamycin + aldosterone, chloroquine, chloroquine + aldosterone, 3-MA or 3-MA + aldosterone for $2 \mathrm{~h}$. Subsequently, the green fluorescent puncta of 50 cells in each group were counted (Fig. 1D). Western blot analysis demonstrated that aldosterone promoted the expression of Beclin-1 and the conversion of LC3-I to LC3-II in the NRK-52E cells (Fig. 1E and F).

Aldosterone induces autophagy through the generation of ROS. The NRK-52E cells were stained with acridine orange to investigate the formation of aldosterone-induced autophagic vacuoles (Fig. 2A and B). Under confocal microscopy, the number of autophagic vacuole was significantly increased in the cells treated with aldosterone, rapamycin, and rapamycin + aldosterone. Notably, fewer green fluorescent puncta were observed following incubation with 3-MA and NAC, compared with the aldosterone-induced cells. Western blot analysis demonstrated that the expression of Beclin-1 and LC3-II were inhibited in the cells treated with NAC (Fig. 2C and E). Western blot analysis was also performed to detect the expression levels of SOD2 and catalase. The cells treated with aldosterone promoted the expression levels of SOD2 and catalase, inversely, the expression levels of SOD2 and catalase were inhibited in the NAC-pretreated cells (Fig. 2D and F).

To determine the levels of oxidative stress in the aldosterone-treated cells, the cells were stained with DCFH-DA, which is oxidized to DCF by intracellular ROS, and the fluorescence of the DCF was measured using flow cytometry (Fig. 3). The results revealed that treatment with NAC markedly reduced the generation of aldosterone-induced ROS. Rosup was used as a positive control. Taken together, these findings suggested that cells treated with NAC repressed the aldosterone-induced autophagy by inhibiting the generation of ROS.

Effects of Rg1 on aldosterone-induced autophagy in NRK-52E cells. To investigate the effects of Rg1 on aldosterone-induced autophagy, the NRK-52E cells were pre-treated with control (buffer), aldosterone, Rg1 and Rg1 + aldosterone, and the proteins were isolated from these cells to perform western blot analysis for catalase, SOD2, LC3B and Beclin-1. The results demonstrated that cells incubated with aldosterone showed higher protein levels of SOD2 and catalase, compared with the control cells, and the cells treated with Rg1 exhibited lower protein levels of SOD2 and catalase following pre-treatment with aldosterone, compared with the cells treated with aldosterone only. This indicated that Rg1 inhibited the generation of ROS. In addition, the protein levels of LC3-II and Beclin-1 were significantly increased in the aldosterone-treated cells, compared with the control, however, when the cells incubated with $\mathrm{Rg} 1$ following aldosterone treatment, the protein levels of LC3-II and Beclin-1 decreased (Fig. 4). These data suggested that Rg1 relieved aldosterone-induced autophagy by inhibiting the generation of ROS in the NRK-52E cells.

Rgl inhibits aldosterone-induced autophagy in NRK-52E cells by regulating the AMPK/mTOR signaling pathway. To investigate the signaling pathway involved in the inhibition of 
A

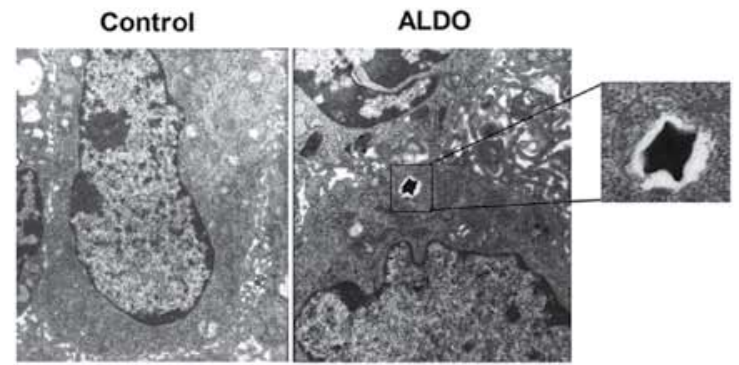

B

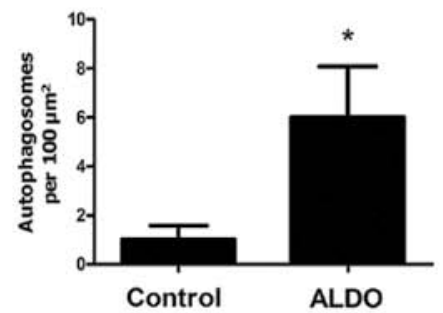

C

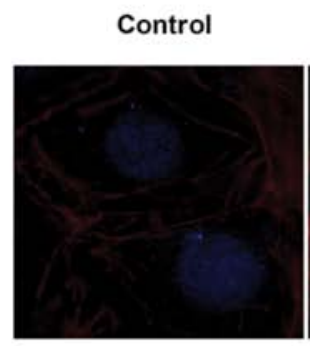

$\mathrm{CL}$

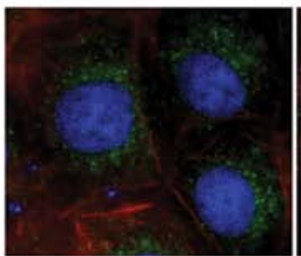

D

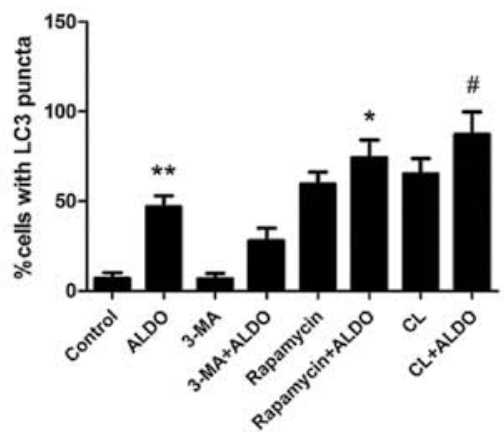

F
ALDO

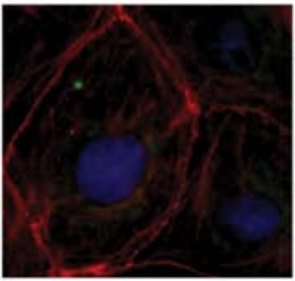

CL+ALDO

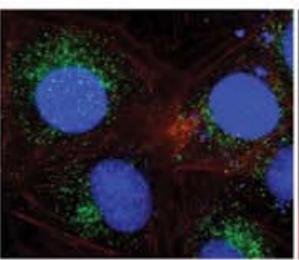

Rapamycin

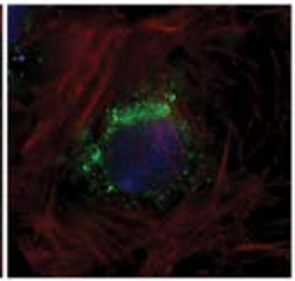

3-MA

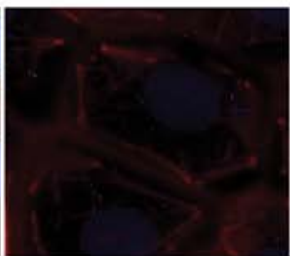

Rapamycin+ALDO

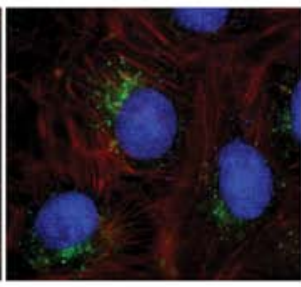

3-MA+ALDO

E
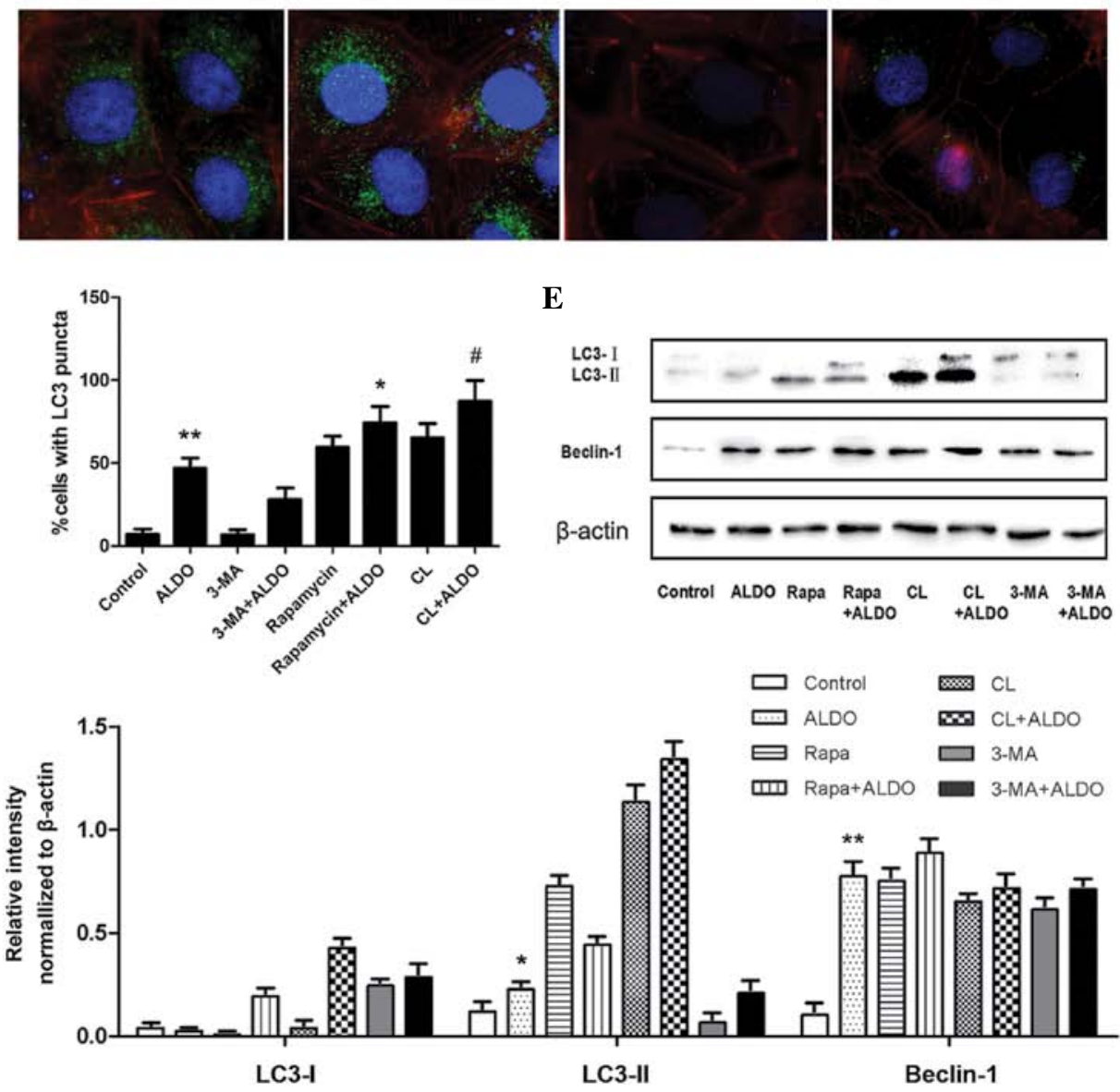

Figure 1. Aldosterone promotes autophagy in NRK-52E cells. (A) Cells were treated with buffer (control) or $10 \mathrm{nmol} / \mathrm{l}$ aldosterone for $12 \mathrm{~h}$, respectively. Under a transmission electron microscope (original magnification, $\mathrm{x} 10,000$ ), increased numbers of autophagosomes were observed in the ALDO-treated cells. (B) Comparison of the number of autophagosomes between the control and ALDO-treated cells, 20 cells were counted in each group $(n=3)$. ${ }^{*}<0.05$, vs. control. (C) Immunofluorescence analysis of the expression of LC3-II in NRK-52E cells exposed to drugs for $12 \mathrm{~h}$. Green fluorescent puncta represent autophagosomes. Images were captured under fluorescence microscopy (magnification, x630). (D) Percentage of autophagosomes with green-fluorescent puncta in each group (50 cells/group; $n=3$ ). ${ }^{* *} \mathrm{P}<0.01$, vs. control. ${ }^{*} \mathrm{P}<0.05$ vs. rapamycin. ${ }^{~} \mathrm{P}<0.05$ vs. CL. (E) Western blot analysis to determine the protein levels of LC3-I, LC3-II and Beclin-1. (F) Quantification of protein levels normalized to $\beta$-actin. ${ }^{*} \mathrm{P}<0.05$, vs. control in the LC3-II group. ${ }^{* *} \mathrm{P}<0.01$, vs. control in the Beclin-1 group. Data are expressed as the mean \pm standard deviation. ALDO, aldosterone; 3-MA, 3-Methyladenine; CL, chloroquine; Rapa, rapamycin.

autophagy by $\operatorname{Rg} 1$, the presents study performed western blot analysis for phosphorylated (p)-AMPK and AMPK (Fig. 5A). Proteins were isolated from the cells following incubation with buffer, aldosterone, aldosterone + Compound $\mathrm{C}$ or aldosterone $+\mathrm{Rg} 1$ for $12 \mathrm{~h}$. Following pre-treatment with aldosterone, the expression of p-AMPK in the NRK-52E cells was 

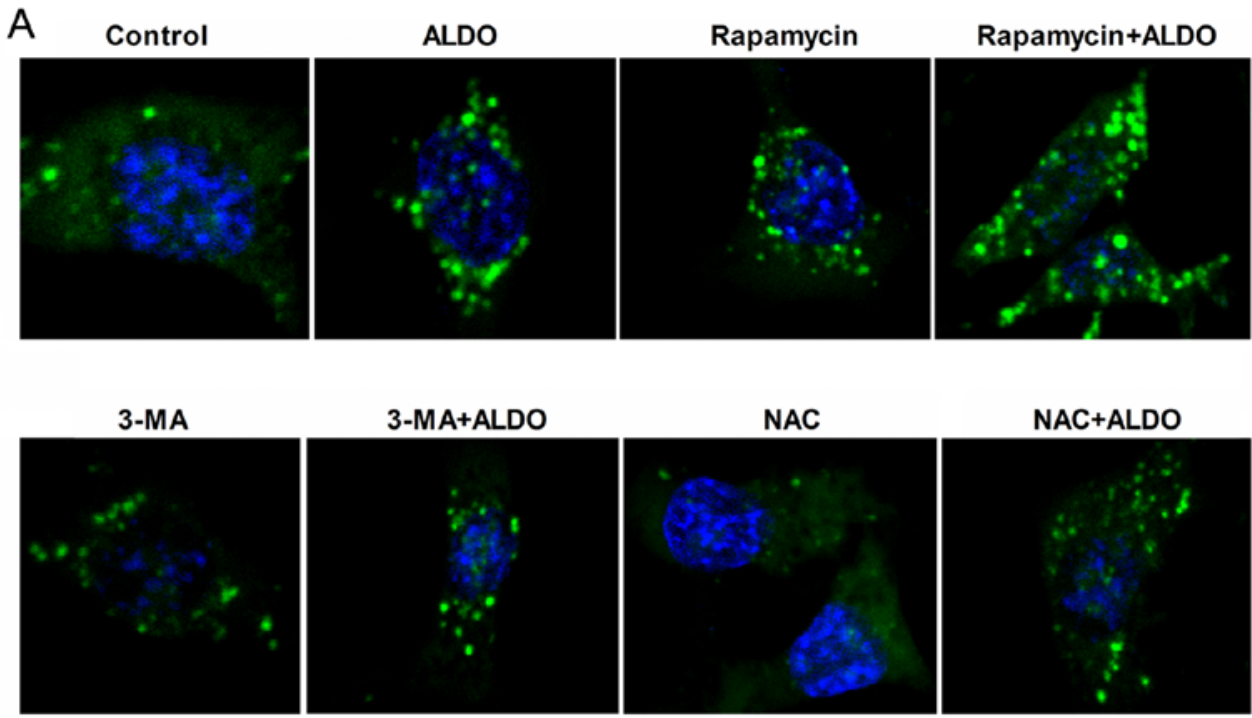

3-MA+ALDO

NAC
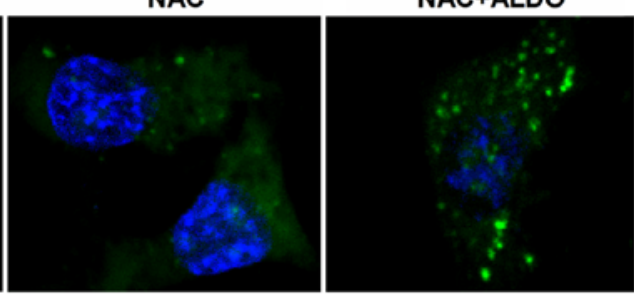

B

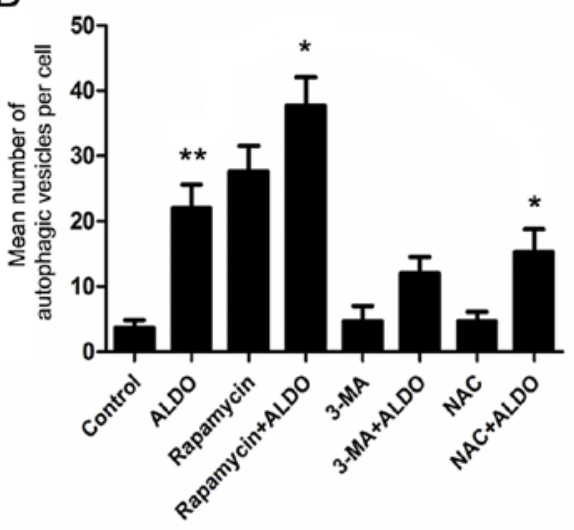

C Beclin-1

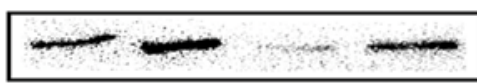

LC3-I

LC3-II

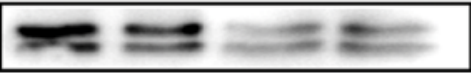

$\beta$-actin

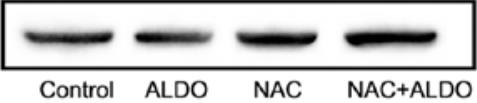

D

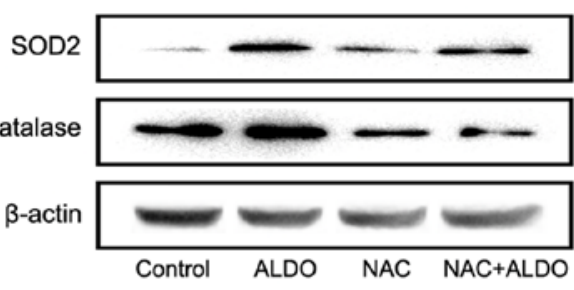

E

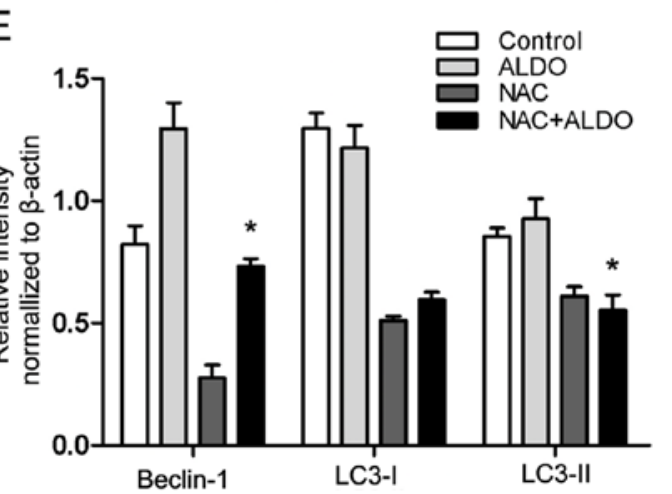

F

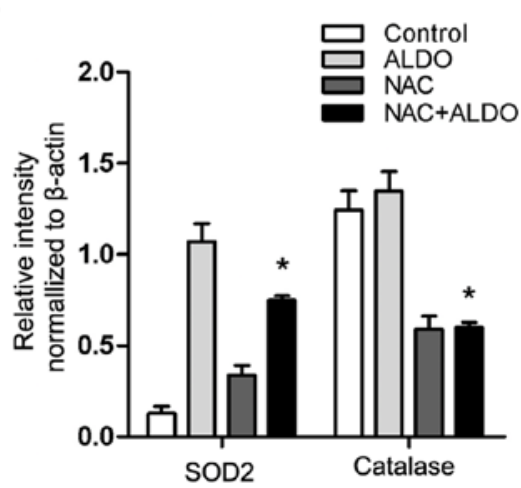

Figure 2. Aldosterone-induced autophagy is reduced through inhibition of reactive oxygen species generation. (A) NRK-52E cells were incubated with acridine orange $(0.05 \mu \mathrm{g} / \mathrm{ml})$ and Hoechst $33342(1 \mu \mathrm{g} / \mathrm{ml})$ for $15 \mathrm{~min}$ following drug treatment for $12 \mathrm{~h}$, and were observed using confocal microscopy. Green fluorescent puncta represent autophagic vacuoles (magnification, $\mathrm{x} 800$ ). (B) Number of autophagic vacuoles in each group (50 cells/group; $n=3)$. " $\mathrm{P}<0.01$, vs. control. ${ }^{*} \mathrm{P}<0.05$, vs. aldosterone. (C) Western blot analysis to detect the protein levels of Beclin-1, LC3-I and LC3-II in NRK-52E cells exposed for $12 \mathrm{~h}$ to buffer (control), ALDO, NAC or NAC + ALDO. (D) Western blot analysis to detect the protein levels of SOD2 and catalase. (E) Quantification of protein levels of Beclin-1, LC3-I and LC3-II, normalized to $\beta$-actin. "P<0.05, vs. aldosterone in each group. (F) Quantification of protein levels of SOD2 and catalase, normalized to $\beta$-actin. "P<0.05, vs. aldosterone in each group. Data are expressed as the mean \pm standard deviation. ALDO, aldosterone; 3-MA, 3-Methyladenine; NAC, N-acetylcysteine; SOD2, superoxide dismutase 2.

significantly increased, compared with the control, however, there was no significant change in the expression of AMPK, suggesting that aldosterone may induce the phosphorylation of AMPK. However, the expression of p-AMPK was reduced to normal levels following incubation of the aldosterone-treated cells with Compound $\mathrm{C}$, which is an inhibitor of AMPK. In addition, the expression of p-AMPK was also reversed in the aldosterone-treated cells by $\operatorname{Rg} 1$, indicating that $\mathrm{Rg} 1$ may reduce the aldosterone-induced phosphorylation of AMPK. The ratio of p-AMPK/AMPK was calculated to measure the 

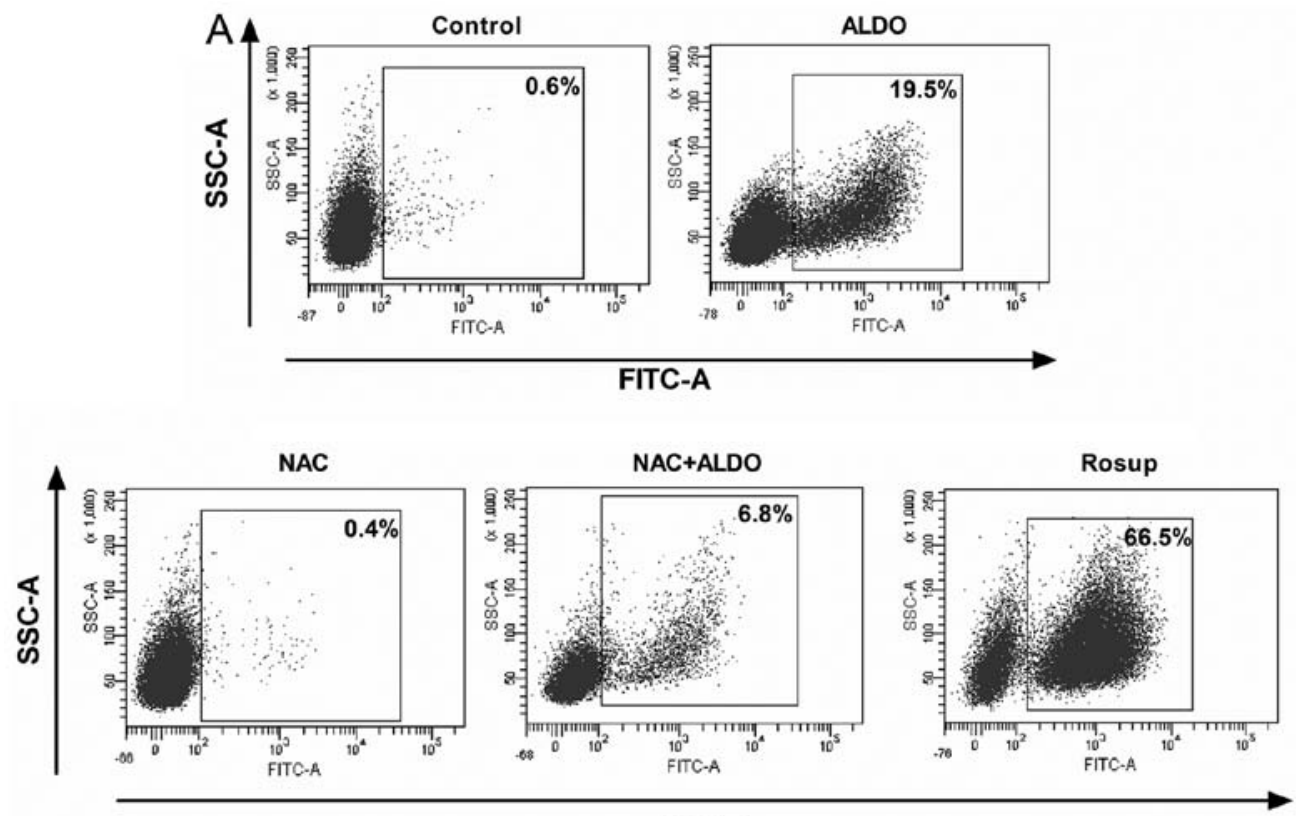

FITC-A

B

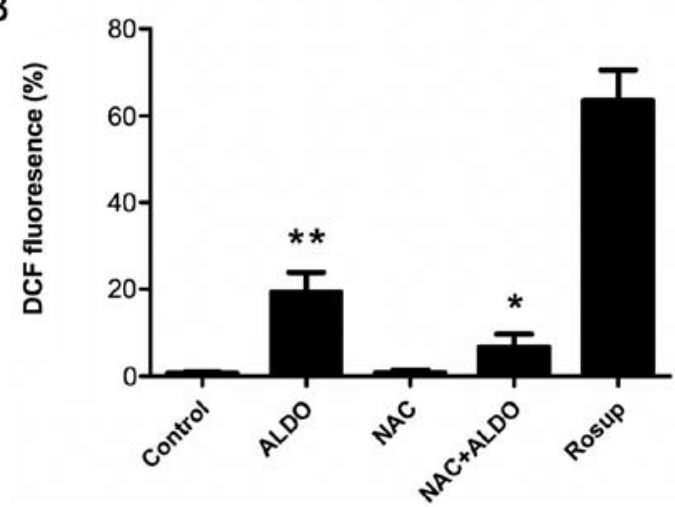

Figure 3. Effects of NAC on aldosterone-induced reactive oxygen species generation in NRK-52E cells. (A) Flow cytometry for DCF fluorescence following DCFH-DA staining. NRK-52E cells were incubated with the drugs for $12 \mathrm{~h}$ prior to performing flow cytometry. Rosup was used as a positive control. (B) Quantification of the DCG fluorescence. Results are expressed as the mean \pm standard deviation $(\mathrm{n}=3){ }^{* * *} \mathrm{P}<0.01$, vs. control; " $\mathrm{P}<0.05$, vs. aldosterone. ALDO, aldosterone; NAC, N-acetylcysteine; FITC, fluorescein isothiocyanate; SSC-A, side scatter area; DCF, 2,7-dichlorofluorescin.

phosphorylation of AMPK (Fig. 5B). It was previously demonstrated that activated AMPK induces autophagy in cells by inhibiting the activation of mTOR and phosphorylation of p70 ribosomal S6 protein kinase (P70S6K), which acts as an important downstream target protein in the mTOR signaling pathway (25). Therefore, the present study performed western blot analysis for p-P70S6K and P70S6K (Fig. 5C). Proteins were harvested from the cells following pre-treatment with buffer, aldosterone, $\mathrm{Rg} 1$ and aldosterone $+\mathrm{Rg} 1$ for $24 \mathrm{~h}$. The results demonstrated that the expression of p-P70S6K was markedly reduced in the aldosterone-treated cells, compared with the control, and incubation of the cells with aldosterone and $\mathrm{Rg} 1$ reversed the reduced expression of p-P70S6K to normal, suggested that Rg1 recovered the phosphorylation of P70S6K, which reduced by aldosterone. The p-P70S6K/P70S6K ratio was calculated to measure the phosphorylation of P70S6K (Fig. 5D). Taken together, these data suggested that $\operatorname{Rg} 1$ inhibited aldosterone-induced autophagy through regulation of the AMPK/mTOR signaling pathway in NRK-52E cells.

\section{Discussion}

In the present study, NRK-52E cells were exposed to aldosterone to examine the potential for aldosterone to induce autophagy in renal tubular epithelial cells, and to investigate the association between aldosterone-induced autophagy and ROS generation, as well as the effects of $\mathrm{Rg} 1$ on this process. Initially, increased autophagosomes and elevated conversion of LC3-I to LC3-II were observed in the aldosterone-treated NRK-52E cells, suggesting enhanced autophagy in these cells, which was further validated using acridine orange staining, flow cytometry and western blot analysis. Furthermore, increased ROS generation was observed to mediat aldosterone-induced autophagy and this was reversed by $\mathrm{Rg} 1$, possibly through regulation of the AMPK/mTOR signaling pathway.

Aldosterone is involved in various renal pathologies, including glomerulosclerosis, proteinuria and fibrosis (26). A number of previous studies investigating the role of aldosterone in renal diseases have revealed the importance of aldosterone in the processes of inflammation and fibrosis $(27,28)$. Furthermore, 


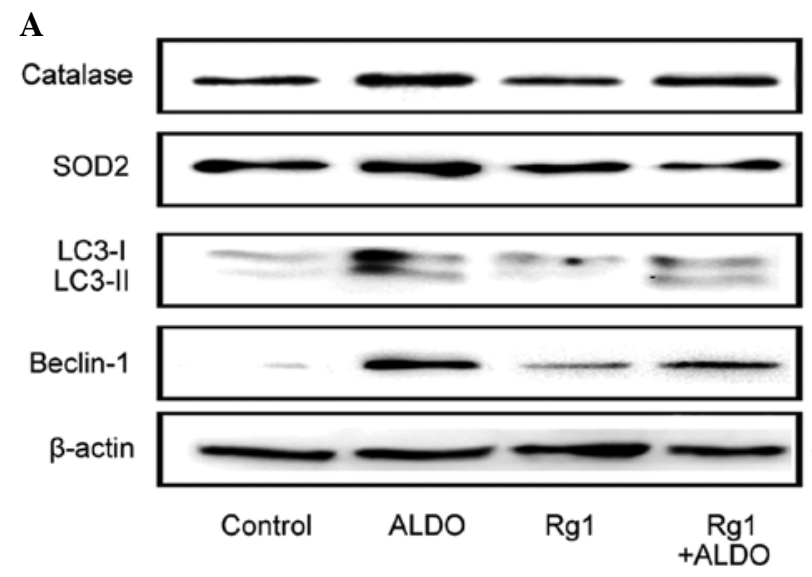

$\mathbf{B}$

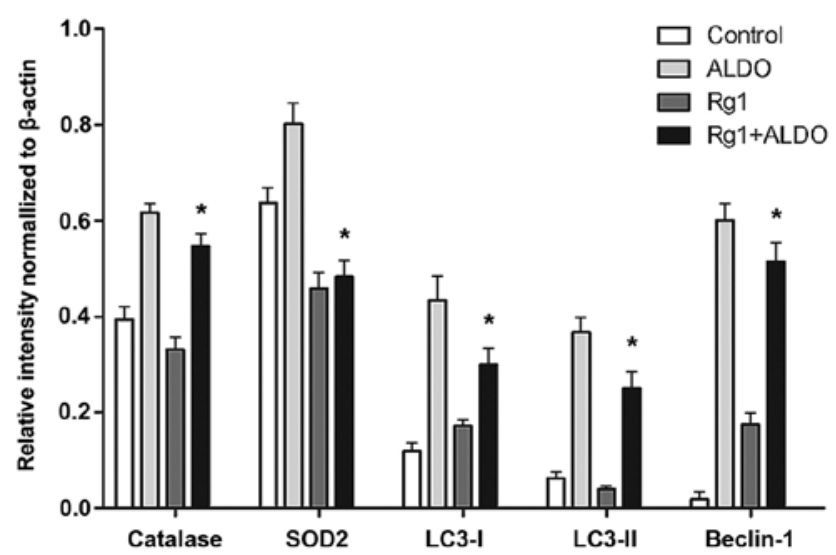

Figure 4. Effects of Rg1 on aldosterone-induced autophagy in NRK-52E cells. (A) Western blot analysis to detect the protein levels of catalase, SOD2, LC3-I, LC3-II and Beclin-1. Proteins were isolated from cells following pre-treatment with buffer (control), ALDO, Rg1 or Rg1 + ALDO. (B) Quantification of protein levels normalized to $\beta$-actin. " $\mathrm{P}<0.05$, vs. aldosterone in each group. Data are expressed as the mean \pm standard deviation. ALDO, aldosterone; SOD2, superoxide dismutase 2; Rg1, ginsenoside-Rg1.
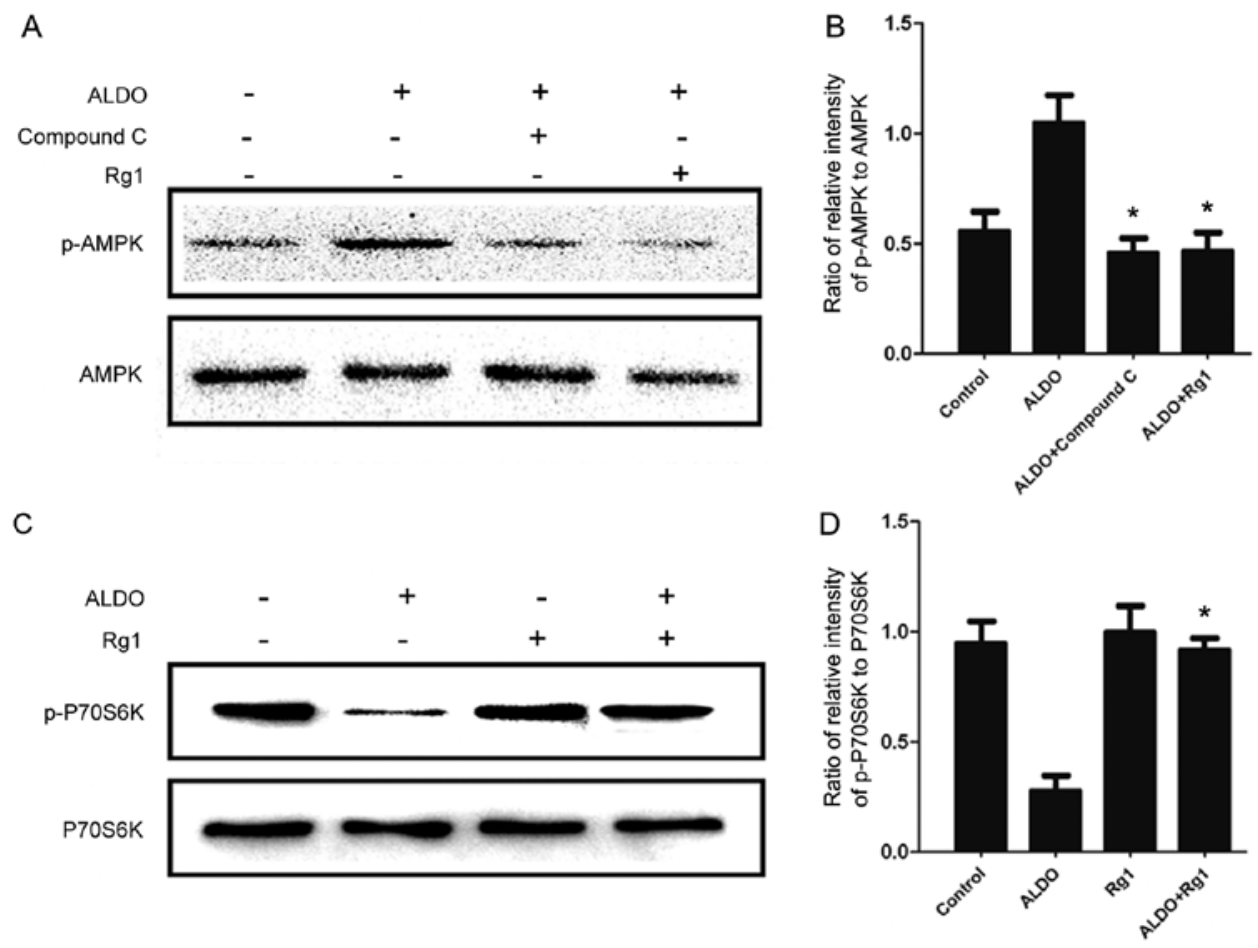

Figure 5. Rg1 inhibits aldosterone-induced autophagy in NRK-52E cells by regulating the AMPK/mTOR signaling pathway. (A) Western blot analysis to detect the protein levels of p-AMPK and AMPK. Proteins were isolated from cells following pre-treatment with buffer (control), ALDO, ALDO + Compound C or ALDO + Rg1, (B) Value of p-AMPK/AMPK. "P<0.05, vs. aldosterone. (C) Western blot analysis to detect the protein levels of p-P70S6K and P70S6K. Proteins were isolated from the cells following pretreatment with buffer (control), ALDO, Rg1 or ALDO + Rg1. (D) Value of p-P70S6K/P70S6K. "P<0.05, vs. aldosterone. Data are expressed as the mean \pm standard deviation.ALDO, aldosterone; SOD2, superoxide dismutase 2; Rg1, ginsenoside-Rg1; P70S6K, p70 ribosomal S6 protein kinase; p-P70S6K, phosphorylated P70S6K.

these reports also demonstrated that aldosterone promotes the production of ROS via MR-dependent mechanisms, and that inhibiting ROS synthesis can prevent the progression of proteinuria and ameliorate renal injury, suggesting the importance of oxidative stress in aldosterone-induced renal injury. However, accumulating evidence has suggested that ROS is important for the regulation of autophagy. Thus, the present study hypothesized that aldosterone promotes renal injury through inducing autophagy via the generation of ROS.
Our previous report demonstrated that aldosterone induces podocyte autophagy through the ROS signaling pathway (24). In the present study, to further investigate the importance of aldosterone in renal injury, aldosterone-induced autophagy was examined in the NRK-52E renal tubular cell line, which is another important cell in kidney.

To investigate the effects of aldosterone in NRK-52E cells, the cells were exposed to $10 \mathrm{nmol} / \mathrm{l}$ aldosterone, and the results revealed that the formation of autophagosomes was notably 
increased in cells treated with aldosterone, compared with the control cells. Consistent with previous reports, chloroquine and rapamycin markedly promoted autophagy in the aldosterone-treated cells in the present study $(23,24)$. Chloroquine is a lysosomal inhibitor, which inhibits lysosome-autophagosome fusion, and rapamycin is an inhibitor of mTOR. To investigate the formation of autophagosomes, the present study measured the LC3-II, the protein levels of which in cells represent the dynamic balance between the production and degradation of autophagosomes (13). The results revealed that the expression of LC3-II was elevated in aldosterone-treated cells. In addition, the expression of Beclin-1, which is involved in the formation of autophagosomes, was markedly increased in these cells. In the present study, the results of the immunofluorescence analysis demonstrated that the effects on the cells treated with aldosterone were the same effects as those exposed to chloroquine or rapamycin, and further enhanced aldosterone-induced autophagy. By contrast, 3-MA inhibited the formation of autophagosome in the aldosterone-treated cells. These results suggested that aldosterone promoted autophagy in the NRK-52E cells.

To elucidate the role of antioxidation in autophagy in the present study, the NRK-52E cells were treated with the antioxidant, NAC, following incubation with aldosterone, which resulted in the formation of fewer autophagosomes in the NAC + aldosterone treatment group, compared with the aldosterone treatment group, assessed by staining with acridine orange. Further investigation demonstrated that cellular antioxidative enzymes, including SOD2 and catalase, were increased in aldosterone-treated cells, indicatinga protective mechanism against ROS-mediated injury. In addition, the protein levels of SOD2 and catalase were notably decreased in the cells treated with NAC, which also inhibited the expression of Beclin-1 and LC3-II. Results of the flow cytometry also confirmed the increased oxidation in the aldosterone-treated cells, which was reversed by NAC. Therefore, it was hypothesized that inhibiting the generation of ROS can reduce aldosterone-induced autophagy.

Due to the property of antioxidants, ginsenoside-Rg1 was used to inhibit the generation of ROS, thereby attempting to reduce aldosterone-induced autophagy in the NRK-52E cells. Previous reports have already demonstrated the role of $\mathrm{Rg} 1$ in inhibiting autophagy in hypoxia/reoxygenation-treated H9c2 cardiomyocytes. The results of the present study revealed that the cells pretreated with $\mathrm{Rg} 1$ reduced the expression of SOD2, catalase, Beclin-1 and LC3-II. In addition, Rg1 significantly inhibited aldosterone-induced autophagy. To investigate the signaling pathways involved in Rg1 inhibiting autophagy, the expression levels of AMPK and p-AMPK were examined. AMPK is a major regulator of energy homeostasis, and adenosine triphosphate depletion can activate AMPK to decompose fatty acids and induce autophagy (29). In addition, AMPK can activate TSC1/2, and inhibit Rheb and mTORC1, promoting autophagy. P70S6K, is a downstream serine/threonine kinase of mTOR, and activated mTOR is involved in several cellular functions by phosphorylating specific factors, including P70S6K (30). In the present study, Rg1 inhibited the activation of AMPK by reducing the phosphorylation of AMPK and increasing the expression of p-P70S6K in the NRK-52E cells, which revealed that the activation of $\mathrm{Rg} 1$ may involve in AMPK/ mTOR signaling pathway.

In conclusion, the present study demonstrated that aldosterone induced autophagy via the generation of ROS in NRK-52E cells. Rg1 notably relieved oxidative stress in the aldosterone-pre-treated NRK-52E cells and inhibited the activation of AMPK to sustain mTOR activity, thereby markedly reducing aldosterone-induced autophagy. These findings provide novel insight into the protective effects of $\mathrm{Rg} 1$ in renal tubular cells and may assist in developing novel treatments for kidney diseases.

\section{Acknowledgements}

The present study was supported by grants from the National Natural Science Foundation of China (grant. no. 81170667), the Youth Science and Technology Support Program of Sichuan Province (grant. no. 2011JDT0014), the Education Department Program of Sichuan Provine (grant. no. 15ZA0253) and the Chengdu Medical College Program (grant. no. CYZ14-011).

\section{References}

1. Crabbe J: Stimulation of active sodium transport by the isolated toad bladder with aldosterone in vitro. J Clin Invest 40: 2103-2110, 1961.

2. Nguyen Dinh Cat A and Jaisser F: Extrarenal effects of aldosterone. Curr Opin Nephrol Hypertens 21: 147-156, 2012.

3. McGowan AJ, Fernandes RS, Samali A and Cotter TG: Anti-oxidants and apoptosis. Biochem Soc Trans 24: 229-233, 1996.

4. Patni H, Mathew JT, Luan L, Franki N, Chander PN and Singhal PC: Aldosterone promotes proximal tubular cell apoptosis: Role of oxidative stress. Am J Physiol Renal Physiol 293: F1065-F1071, 2007.

5. Nishiyama A and Abe Y: Aldosterone and renal injury. Nippon Yakurigaku Zasshi 124: 101-109, 2004.

6. Shibata S, Nagase M, Yoshida S, Kawachi H and Fujita T: Podocyte as the target for aldosterone: Roles of oxidative stress and Sgk1. Hypertension 49: 355-364, 2007.

7. Queisser N, Amann K, Hey V, Habib SL and Schupp N: Blood pressure has only minor influence on aldosterone-induced oxidative stress and DNA damage in vivo. Free Radic Biol Med 54: 17-25, 2013.

8. Yu L: Recent progress in autophagy. Cell Res 24: 1-2, 2014.

9. Ravikumar B, Sarkar S, Davies JE, Futter M, Garcia-Arencibia M, Green-Thompson ZW, Jimenez-Sanchez M, Korolchuk VI, Lichtenberg M,Luo S, et al: Regulation of mammalian autophagy in physiology and pathophysiology. Physiol Rev 90: 1383-1435, 2010.

10. Mizushima N, Levine B, Cuervo AM and Klionsky DJ: Autophagy fights disease through cellular self-digestion. Nature 451: 1069-1075, 2008.

11. Chen Y and Gibson SB: Is mitochondrial generation of reactive oxygen species a trigger for autophagy? Autophagy 4: 246-248, 2008.

12. Xie Z and Klionsky DJ: Autophagosome formation: Core machinery and adaptations. Nat Cell Biol 9: 1102-1109, 2007.

13. Kabeya Y, Mizushima N, Ueno T, Yamamoto A, Kirisako T, Noda T, Kominami E, Ohsumi Y and Yoshimori T: LC3, a mammalian homologue of yeast Apg8p, is localized in autophagosome membranes after processing. EMBO J 19: 5720-5728, 2000.

14. Kihara A, Kabeya Y, Ohsumi Y and Yoshimori T: Beclin-phosphatidylinositol 3-kinase complex functions at the trans-Golgi network. EMBO Rep 2: 330-335, 2001.

15. Furuya N, Yu J, Byfield M, Pattingre S and Levine B: The evolutionarily conserved domain of Beclin 1 is required for Vps34 binding, autophagy and tumor suppressor function. Autophagy 1: 46-52, 2005.

16. Itakura $\mathrm{E}$, Kishi $\mathrm{C}$, Inoue $\mathrm{K}$ and Mizushima $\mathrm{N}$ : Beclin 1 forms two distinct phosphatidylinositol 3-kinase complexes with mammalian Atg14 and UVRAG. Mol Biol Cell 19: 5360-5372, 2008. 
17. Sun Q, Fan W, Chen K, Ding X, Chen S and Zhong Q Identification of Barkor as a mammalian autophagy-specific factor for Beclin 1 and class III phosphatidylinositol 3-kinase. Proc Natl Acad Sci USA 105: 19211-19216, 2008.

18. Butler D, Brown QB, Chin DJ, Batey L, Karim S, Mutneja MS, Karanian DA and Bahr BA: Cellular responses to protein accumulation involve autophagy and lysosomal enzyme activation. Rejuvenation Res 8: 227-237, 2005.

19. Lü JM, Yao Q and Chen C: Ginseng compounds: An update on their molecular mechanisms and medical applications. Curr Vasc Pharmacol 7: 293-302, 2009.

20. Liu CX and Xiao PG: Recent advances on ginseng research in China. J Ethnopharmacol 36: 27-38, 1992.

21. Mochizuki M, Yoo YC, Matsuzawa K, Sato K, Saiki I, Tono-oka S, Samukawa K and Azuma I: Inhibitory effect of tumor metastasis in mice by saponins, ginsenoside-Rb2, 20(R)and 20(S)-ginsenoside-Rg3, of red ginseng. Biol Pharm Bull 18: 1197-1202, 1995.

22. Zhu D, Wu L, Li CR, Wang XW, Ma YJ, Zhong ZY, Zhao HB, Cui J, Xun SF, Huang XL, et al: Ginsenoside Rg1 protects rat cardiomyocyte from hypoxia/reoxygenation oxidative injury via antioxidant and intracellular calcium homeostasis. J Cell Biochem 108: 117-124, 2009.

23. Zhang ZL, Fan Y and Liu ML: Ginsenoside Rg1 inhibits autophagy in $\mathrm{H} 9 \mathrm{c} 2$ cardiomyocytes exposed to hypoxia/reoxygenation. Mol Cell Biochem 365: 243-250, 2012.
24. Mao N, Cheng Y, Shi XL, Wang L, Wen J, Zhang Q, Hu QD and Fan JM: Ginsenoside Rg1 protects mouse podocytes from aldosterone-induced injury in vitro. Acta Pharmacol Sin 35: 513-522, 2014

25. Kim J, Kundu M, Viollet B and Guan KL: AMPK and mTOR regulate autophagy through direct phosphorylation of Ulk1. Nat Cell Biol 13: 132-141, 2011.

26. Ibrahim HN, Rosenberg ME and Hostetter TH: Role of the renin-angiotensin-aldosterone system in the progression of renal disease: A critical review. Semin Nephrol 17: 431-440, 1997.

27. Namsolleck P and Unger T: Aldosterone synthase inhibitors in cardiovascular and renal diseases. Nephrol Dial Transplant 29 (Suppl 1): i62-i68, 2014.

28. Nagase M, Yoshida S, Shibata S, Nagase T, Gotoda T, Ando K and Fujita T: Enhanced aldosterone signaling in the early nephropathy of rats with metabolic syndrome: possible contribution of fatderived factors. J Am Soc Nephrol 17: 3438-3446, 2006.

29. Hardie DG: AMP-activated/SNF1 protein kinases: conserved guardians of cellular energy. Nat Rev Mol Cell Biol 8: 774-785, 2007.

30. Xu JT, Zhao X, Yaster M and Tao YX: Expression and distribution of mTOR, p70S6K, 4E-BP1, and their phosphorylated counterparts in rat dorsal root ganglion and spinal cord dorsal horn. Brain Res 1336: 46-57, 2010. 